\title{
GERMINAÇÃO DE SEMENTES DE Caesalpinia pyramidalis Tul. (CATINGUEIRA) SUBMETIDAS A DEFICIÊNCIA HÍDRICA ${ }^{1}$
}

\begin{abstract}
Cimille Gabrielle Cardoso Antunes ${ }^{2}$, Claudineia Regina Pelacani², Renata Conduru Ribeiro ${ }^{2}$, Jonaicon Vieira de Souza ${ }^{2}$, Cintia Luiza Mascarenhas de Souza ${ }^{2}$ e Renato Delmondez de Castro ${ }^{3}$

RESUMO - A disponibilidade hídrica é um dos principais fatores ambientais capaz de influenciar o processo de germinação de sementes. Nessas condições, a habilidade das sementes em manter sua viabilidade durante o armazenamento pode ser uma vantagem adaptativa de espécies encontradas em diferentes biomas, especialmente na caatinga. Objetivou-se, com este trabalho, avaliar a influência do armazenamento na tolerância das sementes de catingueira (Caesalpinia pyramidalis Tul.) à restrição hídrica, utilizando-se como agente osmótico o polietilenoglicol 6000 (PEG 6000). Sementes de catingueira foram armazenadas por 0, 3 e 6 meses em temperatura ambiente e acondicionadas em sacos de papel kraft, avaliando-se a influência dos diferentes potenciais osmóticos $(0,0 ;-0,2 ;-0,4 ;-0,6 ;-0,8$; -1,0 e -1,2 MPa) das soluções de PEG 6000 sobre a porcentagem de germinação (G\%), no tempo médio de germinação (Tm), na velocidade média de germinação (Vm) e no índice de velocidade de germinação (IVG) das sementes. Observou-se que a interação entre o armazenamento e os tratamentos osmóticos influenciou significativamente as variáveis cinéticas de germinação avaliadas, exceto a Vm. Em todos os meses avaliados, a redução da água disponível no meio afetou no decréscimo da G\%, da Vm e do IVG e na elevação do Tm das sementes. A tolerância à deficiência hídrica nas sementes de catingueira foi relativamente alta, tendo como limite a faixa entre -0,8 e -1,0 MPa. A identificação dos fatores ambientais que governam a germinação das sementes, como a disponibilidade hídrica, representa uma importante ferramenta na interpretação do comportamento ecológico das espécies em hábitats naturais.
\end{abstract}

Palavras-chave: Catingueira, Tolerância, Germinação e PEG 6000.

\section{GERMINATION OF Caesalpinia pyramidalis TUL. SEEDS (CATINGUEIRA) UNDER WATER DEFICIT}

\begin{abstract}
Water availability is one of the main environmental factor able to influence the germinative process of seeds. In these conditions, the ability of seeds to maintain their viability during the storage can be an adaptive advantage of species found in differents biomes, especially in the Caatinga. The objective of this work was to assess the influence of storage on tolerance of seed of catingueira (Caesalpinia pyramidalis Tul.) to water restriction, using polyethyleneglycol 6000 (PEG 6000) as osmotic agent. Seeds of catingueira were stored for 0,3 and 6 months at room temperature and packed in kraft paper bags, assessing the influence of different osmotic potentials $(0.0 ;-0.2 ;-0.4 ;-0.6 ;-0.8 ;-1.0$ and $-1.2 \mathrm{MPa})$ of PEG 6000 solutions on percentage of germination ( $G \%)$, average time of germination (AvT), average speed of germination (AvS) and speed rate of germination (SRG) of seeds. It was observed that the interaction between storage and osmotic treatments significantly influenced the evaluated germination kinetic variables, except AvS. In all evaluated months, reduction of available water in the medium affected decrease of $G \%$, AvT and it affected increase of AvT of the seeds. The tolerance to water deficit in the seeds of catingueira was relatively high, and the limit was within the range from -0.8 to -1.0 MPa. The identification of environmental factors that rule seed germination, as the water availability, represents an important tool in the interpretation of the ecological behavior of the species under conditions of natural habitats.
\end{abstract}

Keywords: Catingueira, Tolerance, Germination and PEG 6000.

\footnotetext{
${ }^{1}$ Recebido em 14.07.2009 e aceito para publicação em 02.05.2011.

${ }^{2}$ Laboratório de Germinação de Sementes, UEHF, UEFS, Feira de Santana, BA, Brasil. E-mail: <cimillebio@gmail.com>.

${ }^{3}$ Laboratório de Bioquímica, Biotecnologia e Bioprodutos, ICS, UFBA, Salvador, BA, Brasil.
} 


\section{INTRODUÇÃO}

O nordeste brasileiro representa uma das regiões áridas com território ocupado quase totalmente por espécies xerófilas, que apresentam características morfoanatômicas e fisiológicas especiais, constituindo o bioma Caatinga (SAMPAIO; RODAL, 2000). A Caesalpinia pyramidalis Tul. é uma das espécies amplamente distribuídas na Região Nordeste, no entanto é endêmica da caatinga. Exibe propriedades madeireiras, forrageiras e medicinais, e é muito explorada localmente (FRANCELINO et al., 2003; MONTEIRO et al., 2005).

A caatinga exibe fisionomia desértica, com índices pluviométricos em torno de 500 a $700 \mathrm{~mm}$ anuais e temperaturas que variam de 24 a $26^{\circ} \mathrm{C}$. Além disso, há predominância de vento seco, que contribui para a aridez da paisagem nos meses de seca (SAMPAIO; RODAL, 2000). Os solos possuem um potencial osmótico extremamente negativo e elevadas concentrações de íons potencialmente tóxicos (devido às práticas agrícolas errôneas de irrigação), que contribuem para a retenção de água, indisponibilizando-a para os vegetais (SAMPAIO, 1995; DANTAS et al., 2002).

A disponibilidade hídrica é um dos importantes fatores ambientais capaz de influenciar o processo de germinação de sementes e o estabelecimento das plântulas, visto que os vegetais são geralmente mais sensíveis ao déficit hídrico nas fases iniciais do desenvolvimento (BLAKE, 1993; BARBEDO; MARCOS FILHO, 1998).

A influência da deficiência hídrica na germinação se dá pelo atraso no início do processo ou diminuição na germinação (HARDEGREE; EMMERICH, 1990). Potenciais muito baixos, especialmente no início da embebição das sementes, influenciam na absorção de água, retardando a sequência dos eventos germinativos (BANSAL et al., 1980). Por outro lado, o excesso de umidade em geral provoca decréscimo na germinação, uma vez que impede a difusão do oxigênio e reduz o processo metabólico resultante (BORGES; RENA, 1993).

Uma das técnicas mais difundidas para simular baixa umidade no substrato tem sido o uso de soluções com diferentes potenciais osmóticos (TAYLOR; HARMAN, 1990). O polietilenoglicol (PEG), por ser um carboidrato inerte às células, devido ao seu alto peso molecular (HASEGAWA et al., 1984), tem sido utilizado com sucesso em diversos trabalhos de pesquisa para simular os efeitos da deficiência hídrica nas sementes, determinando o ponto de tolerância à seca em diferentes espécies (SANTOS et al., 1992; BRACCINI et al., 1996; MORAES; MENEZES, 2003).

A tolerância ao estresse hídrico constitui-se em processos complexos e geralmente interligados, envolvendo interações de várias propriedades (ZHU, 2002; FOOLAD, 2004; VERSLUES et al., 2006). Os mecanismos fisiológicos de tolerância à seca têm sido bastante estudados em espécies vegetais durante a fase vegetativa, porém pouco se sabe sobre os mecanismos de adaptação de sementes de espécies nativas às condições de restrição hídrica naturais do semi-árido nordestino. Tendo em vista esses aspectos, propôs-se este trabalho visando avaliar a influência do armazenamento e da deficiência hídrica sobre as características germinativas de sementes de catingueira (Caesalpinia pyramidalis Tul.).

\section{MATERIAL E MÉTODOS}

As sementes foram coletadas em 2007, a partir de matrizes de catingueira localizadas no Município de Petrolina - PE nas seguintes coordenadas geográficas: 9 30' 21' S 40 30' 21'’ W. Segundo a classificação de Köppen, o clima nessa área é caracterizado como tropical semiárido, tipo BshW, seco e quente na parte norte e semiárido quente estípico na parte sul, caracterizado pela escassez e irregularidade das precipitações, com chuvas no verão e forte evaporação em consequência das altas temperaturas (AMBIENTE BRASIL, 2005).

O beneficiamento e o armazenamento das sementes foram feitos no Laboratório de Germinação de Sementes da Unidade Experimental Horto Florestal, da Universidade Estadual de Feira de Santana (UEFS), Feira de Santana, Bahia, Brasil. O beneficiamento consistiu de avaliação visual das sementes, descartando-se as danificadas. Após essa etapa, as sementes foram armazenadas por 0, 3 e 6 meses em sacos de papel kraft (embalagem permeável) na temperatura ambiente de laboratório $\left(25^{\circ} \mathrm{C} \pm 3\right)$, a fim de se verificar o efeito do armazenamento na tolerância das sementes à deficiência hídrica.

Os experimentos foram realizados em gerbox (previamente esterilizados em hipoclorito de sódio concentrado por 15 minutos), contendo duas folhas

Revista Árvore, Viçosa-MG, v.35, n.5, p.1007-1015, 2011

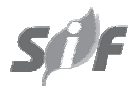


de papel germitest embebidas na solução teste (10 mL). Para simulação do estresse hídrico, foram preparadas soluções de polietilenoglicol (PEG 6000) em diferentes potenciais osmóticos $(0,0 ;-0,2 ;-0,4 ;-0,6 ;-0,8 ;-1,0$ e -1,2 MPa), de acordo com a tabela citada em Villela et al. (1991). A solução de potencial 0,0 MPa foi considerada controle (água destilada). Posteriormente, as sementes foram incubadas em câmara de germinação ajustada à $25^{\circ} \mathrm{C}$, com fotoperíodo de 12 horas, por nove dias. Foram consideradas germinadas as sementes que emergiram radícula acima de $2 \mathrm{~mm}$ de comprimento (foram contadas e descartadas). As variáveis de germinação analisadas foram: porcentagem de germinação (G\%), tempo médio de germinação (Tm), velocidade média de germinação ( $\mathrm{Vm}$ ) e índice de velocidade de germinação (IVG).

O delineamento experimental adotado foi o inteiramente casualizado com quatro repetições de 25 por tratamento, em esquema fatorial $7 \times 3$ (7 potenciais osmóticos $x 3$ períodos de armazenamento). As variáveis respostas foram submetidas à análise de variância e analisadas por regressão ( $\mathrm{p}<0,05)$, através do programa estatístico SISVAR 4.3 (FERREIRA, 1999).

\section{RESULTADOS E DISCUSSÃO}

Observou-se que o déficit hídrico promovido pelas soluções osmóticas de PEG 6000 interferiu, de maneira independente, em todas as variáveis respostas. A porcentagem de sementes germinadas declinou com o aumento da intensidade do estresse hídrico (redução do potencial osmótico). Os meses de armazenamento não interferiram significativamente de maneira independente $(\mathrm{P}<0,05)$ na maior parte das variáveis de germinação avaliadas, apenas quando em interação com os tratamentos osmóticos (Tabela 1).

Os valores da porcentagem de germinação (Figura 1) ajustaram-se ao modelo de regressão quadrático, em todos os meses de estudo (0, 3 e 6 meses), sendo os melhores índices encontrados a -0,2; -0,4 e -0,6 MPa, potenciais osmóticos que simulam baixa deficiência hídrica. Aos 3 e 6 meses, as taxas germinativas nos potenciais supracitados foram superiores, inclusive o controle. Os menores índices de G\% foram encontrados nos potenciais osmóticos mais inferiores (-0,8 e -1,0 MPa), os quais propiciaram condições de estresse mais severas devido à maior concentração de PEG 6000 utilizada.

Apesar de tendência à redução de percentual de germinação com a redução do potencial osmótico do meio, as sementes de catingueira mantiveram a capacidade germinativa em níveis satisfatórios até potenciais de -0,8 a -1,0 MPa. A referida faixa de tolerância encontrase dentro dos padrões observados para outras espécies do semiárido, como Pterogyne nitens Tul. (-1,0 a -1,2 MPa) e Gliricidia sepium (Jacq.) Steud. (-0,8 a -1,0 MPa) (NASSIF et al., 1997; RIBEIRO, 2008), demonstrando que o armazenamento em temperatura ambiente e em saco de papel é efetivo para a manutenção da tolerância à deficiência hídrica em sementes de catingueira em seis meses.

A manutenção da germinação, mesmo com a exposição das sementes 9 dias de déficit hídrico constante e severo, sugere um possível ajustamento osmótico das sementes, envolvendo a acumulação de solutos orgânicos e inorgânicos nas células, em resposta

Tabela 1 - Análise de variância das variáveis cinéticas de germinação das sementes de Caesalpinia pyramidalis Tul. submetidas a diferentes tratamentos de deficiência hídrica e armazenadas por 0, 3 e 6 meses. Feira de Santana, Bahia, agosto de 2007 a fevereiro de 2008.

Table 1 - Analysis of variance of the Kinetic variables of germination of Caesalpinia pyramidalis Tul. seeds subjected to differents treatments of water deficient and stored for 0, 3 and 6 months. Feira de Santana, Bahia, August 2007 to February 2008.

\begin{tabular}{lcccc}
\hline $\begin{array}{l}\text { Fontes de } \\
\text { variação }\end{array}$ & $\begin{array}{c}\text { Germinação } \\
(\mathrm{G} \%)\end{array}$ & $\begin{array}{c}\text { Tempo médio } \\
(\mathrm{Tm})\end{array}$ & $\begin{array}{c}\text { Velocidade } \\
\text { média }(\mathrm{Vm})\end{array}$ & $\begin{array}{c}\text { Velocidade de } \\
\text { Germinação (IVG) }\end{array}$ \\
\hline Meses & $0,0167^{*}$ & $0,5880^{\mathrm{ns}}$ & $0,8987^{\mathrm{ns}}$ & $0,5017^{\mathrm{ns}}$ \\
Tratamentos & $0,0000^{*}$ & $0,0000^{*}$ & $0,0000^{*}$ & $0,0000^{*}$ \\
M x T & $0,0000^{*}$ & $0,0001^{*}$ & $0,1132^{\mathrm{ns}}$ & $0,0000^{*}$ \\
CV\% & 11,83 & 32,97 & 39,79 & 22,70 \\
\hline
\end{tabular}

* = significativo a 5\%; ns = não significativo a 5\%.

$*=$ significant at $5 \%$ probability $; n s=$ not significant at $5 \%$ probability. 


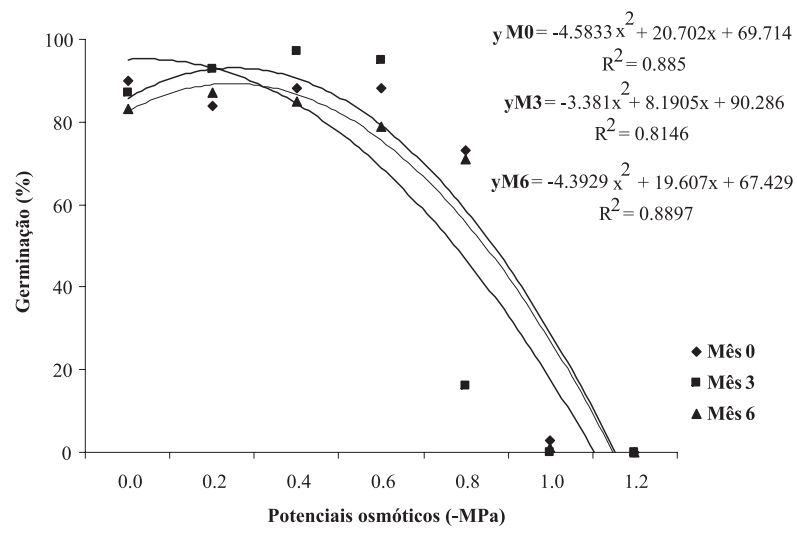

Figura 1 - Germinação (G\%) das sementes de Caesalpinia pyramidalis Tul. submetidas a diferentes tratamentos de deficiência hídrica (-MPa) e armazenadas por 0,3 e 6 meses em temperatura ambiente e saco de papel kraft. Feira de Santana, Bahia, agosto de 2007 a fevereiro de 2008.

Figure 1-Germination (G\%) of the Caesalpinia pyramidalis Tul. seeds subjected to differents treatments of water deficiency $(-M P a)$ and stored for 0,3 and 6 months in room temperature and kraft paper bag. Feira de Santana, Bahia, August 2007 to February 2008.

à queda no potencial hídrico do ambiente celular. Como consequência, o potencial osmótico da célula é diminuído, o que atrai água para o interior celular, garantindo a evolução do processo germinativo (BABU et al., 1999). Tais resultados são relevantes quando se pretende estabelecer mecanismos de sobrevivência de espécies em ambientes áridos, a exemplo da caatinga do nordeste brasileiro.

Perez et al. (2001), avaliando sementes de canafístula (Peltophorum dubium (Spreng.) Taub.) submetidas a déficit hídrico, verificaram elevada germinação nos potenciais osmóticos maiores e redução nos potenciais menores, enquanto que Nassif et al. (1997), estudando o comportamento de sementes de amendoim-do-campo (Pterogyne nitens Tul.) com restrição hídrica, constataram que houve elevada germinação até -0,6 MPa, mas, abaixo desse potencial, a germinação reduziu. Em outro trabalho, Fonseca et al. (2003), analisando sementes de Adenanthera pavonina L. em baixa disponibilidade hídrica, verificaram que a redução do potencial osmótico das soluções de PEG $\left(25^{\circ} \mathrm{C}\right)$ estimulou a queda do percentual de germinação.

Com -1,2 MPa a germinação das sementes foi nula (Figura 1). Quando as sementes embebidas na solução osmótica de -1,2 MPa foram transferidas para água,

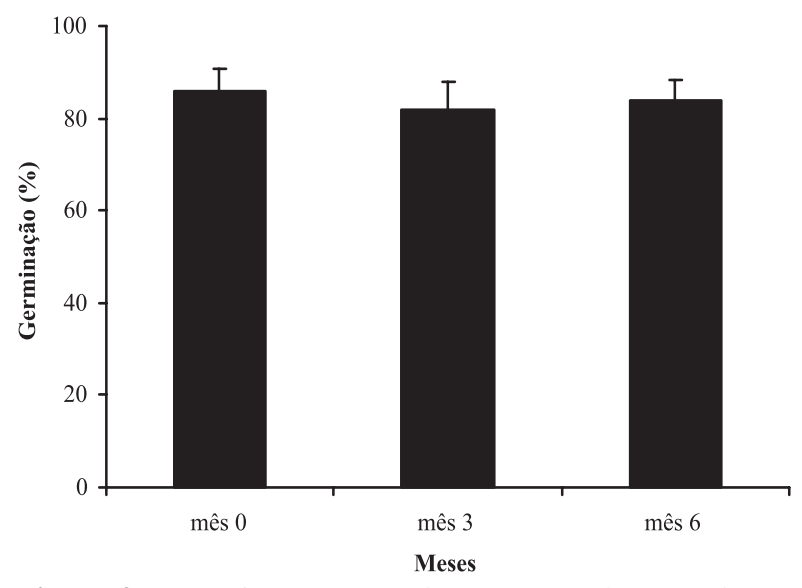

Figura 2 - Germinação (G\%) das sementes de Caesalpinia pyramidalis Tul. em água, após tratamento de estresse osmótico com solução de PEG 6000 a $-1,2 \mathrm{MPa}$, aos 0,3 e 6 meses de armazenamento, em temperatura ambiente e saco de papel kraft. Feira de Santana, Bahia, agosto de 2007 a fevereiro de 2008.

Figure 2 - Germination (G\%) of Caesalpinia pyramidalis Tul. seeds in water, after osmotic stress treatment with PEG 6000 solution at $-1.2 \mathrm{MPa}$, at 0, 3 and 6 months of storage in room temperature and kraft paper bag. Feira de Santana, Bahia, August 2007 to February 2008. 2007 to February 2008.

houve excelente recuperação da germinação no mês zero (86\%), no terceiro (82\%) e no sexto mês (84\%) de armazenamento (Figura 2).

Tal resposta das sementes de catingueira em potenciais extremamente negativos (-1,2 MPa), pode ser atribuída ao fato de que, em condições hídricas muito desfavoráveis, as sementes evitam a germinação como estratégia de sobrevivência, a fim de garantir o estabelecimento de futuras plântulas (BEWLEY; BLACK, 1982; FERREIRA; BORGHETTI, 2004). De acordo com Carvalho e Nakagawa (2000), o potencial hídrico afeta o alongamento celular e a síntese de parede. Para cada espécie, há um valor de potencial hídrico crítico, abaixo do qual a germinação não ocorre(CARVALHO, 2005). Para Bewley e Black(1994), a inibição na emergência da raiz principal decorrente de uma disponibilidade menor de água relaciona-se, frequentemente, a reduções na atividade enzimática e no metabolismo seminal. A recuperação da capacidade germinativa das sementes em condições ótimas pode ser justificada pelo alto nível de tolerância das sementes ao estresse osmótico, corroborando a manutenção da viabilidade e do vigor das sementes durante o armazenamento. 


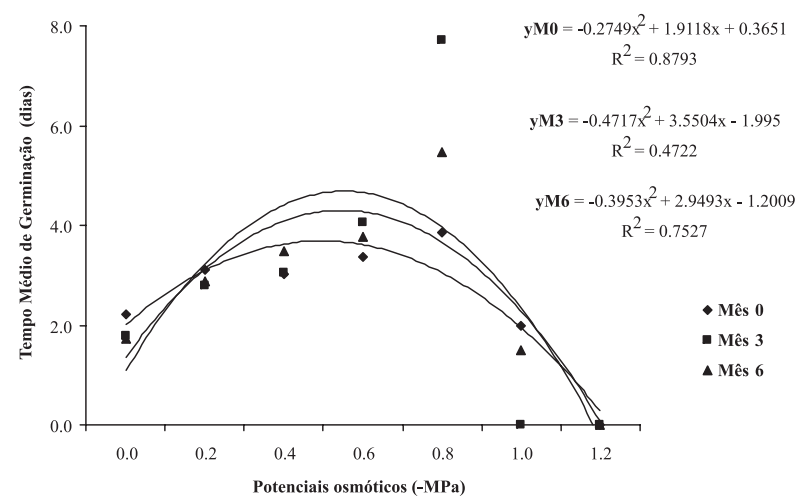

Figura 3 - Tempo médio de germinação (Tm) das sementes de Caesalpinia pyramidalis Tul. submetidas a diferentes tratamentos de deficiência hídrica (-MPa) e armazenadas por 0, 3 e 6 meses em temperatura ambiente e saco de papel kraft. Feira de Santana, Bahia, agosto de 2007 a fevereiro de 2008.

Figure 3-Average Time of germination (AvT) of Caesalpinia pyramidalis Tul. seeds subjected to differents treatments of water deficiency (-MPa) and stored for 0,3 and 6 months in room temperature and kraft paper bag. Feira de Santana, Bahia, August 2007 to February 2008.

Os dados de tempo médio de germinação das sementes (Figura 3) também se ajustaram ao modelo quadrático, no qual se observou que a redução do potencial osmótico ocasionou um atraso no início da germinação das sementes em todos os meses avaliados. $\mathrm{Na}$ faixa compreendida entre -0,4 e-0,8 MPa, verificou-se maior requerimento de dias para que o processo germinativo fosse completado. Quando foram comparadas as sementes armazenadas por 3 e 6 meses, verificou-se que a redução do Tm das sementes nos potenciais -1,0 e -1,2 MPa é justificável pelo efeito do PEG 6000.

O perfil do Tm das sementes seguiu a mesma tendência da germinação, o que pode ser explicado por Hadas (1976), que descreve que mudanças no potencial hídrico externo produzem um efeito nas propriedades hidráulicas do tegumento seminal, e quanto mais baixo for esse potencial, menor será a difusibilidade desse naágua. Isso retardará a absorção de água pela semente, atrasando a emergência da radícula, que caracteriza o fim do processo germinativo. O requerimento de mais dias para que a germinação fosse completada na faixa de $-0,4$ a $-0,8 \mathrm{MPa}$, aos 3 e 6 meses de armazenamento, sugere que, a longo prazo, a tolerância das sementes à deficiência hídrica pode ser comprometida se forem mantidas em embalagem permeável. Jeller et al. (2003), estudando o comportamento de sementes de Cassia excelsa sob estresse hídrico, simulado com soluções de PEG 6000, também verificaram aumento no tempo médio de germinação das sementes quando o potencial osmótico foi reduzido de -0,2 para -0,8 $\mathrm{MPa}$.

Os índices referentes à velocidade média de germinação (Figura 4) foram mais bem ajustados ao modelo de regressão linear, os quais foram influenciados somente pelos tratamentos osmóticos. A variável Vm das sementes de catingueira mostrouse mais sensível à deficiência hídrica do que a germinabilidade, haja vista que, a partir do potencial -0,2 MPa, já se observou uma diminuição dos valores, enquanto para a germinação houve redução somente a partir do potencial -0,8 MPa. Tal perfil corresponde a uma tendência geral dos dados.



Figura 4 - Velocidade média de germinação (Vm) das sementes de Caesalpinia pyramidalis Tul. submetidas a diferentes tratamentos de deficiência hídrica (-MPa) e armazenadas por 0 , 3 e 6 meses em temperatura ambiente e saco de papel kraft. Feira de Santana, Bahia, agosto de 2007 a fevereiro de 2008.

Figure 4-Germination average speed (AvS) of Caesalpinia pyramidalis Tul. seeds subjected to differents treatments of water deficiency (-MPa) and stored for 0,3 and 6 months in room temperature and kraft paper bag. Feira de Santana, Bahia, August 2007 to February 2008.

Revista Árvore, Viçosa-MG, v.35, n.5, p.1007-1015, 2011 
A redução da velocidade média de germinação das sementes de catingueira diante da redução do potencial osmótico pode ser explicada pelo efeito do PEG 6000 na embebição das sementes, uma vez que, por sua alta viscosidade e peso molecular, retarda a velocidade de hidratação dos tecidos e a difusibilidade de oxigênio, permitindo maior tempo para a reorganização das membranas e desenvolvimento de processos metabólicos. Assim, como a resposta das sementes à deficiência hídrica é heterogênea, a germinação é distribuída no tempo e no espaço, permitindo que, em condições naturais, as plântulas encontrem condições ambientais adequadas ao seu estabelecimento e desenvolvimento inicial (WOODSTOCK; TAO, 1981; BEWLEY; BLACK, 1985; 1994).

Nas mesmas condições desse estudo, outros autores também verificaram maior sensibilidade da Vm ao estresse hídrico do que a G\%. Cavalcante et al. (1995) observaram redução na velocidade média de germinação de sementes de Leucaena leucocephala a partir de -0,3 MPa, enquanto a porcentagem de germinação só foi reduzida a partir de -0,6 MPa. Nassif et al. (1997), estudando o amendoim-do-campo, também observaram que a velocidade média de germinação das sementes foi mais afetada pelo déficit hídrico (com o PEG 6000) do que a capacidade germinativa. Neste estudo, houve redução significativa a partir de -0,4 MPa, enquanto para a germinação essa redução só ocorreu a partir de -0,6 MPa. No entanto, Fonseca et al. (2003) observaram redução em ambas as variáveis (germinação e velocidade média de germinação) a partir do mesmo potencial osmótico (-0,1 MPa) em sementes de Adenanthera pavonina.

Os dados de IVG das sementes recém-colhidas (Figura 5) se ajustaram ao modelo quadrático, sendo estes índices elevados em potenciais maiores (-0,2 a -0,6 MPa) e com tendência a declínio em potenciais menores (-0,8; -1,0 e -1,2 MPa). Os dados de IVG das sementes aos 3 e 6 meses de armazenamento, no entanto, se ajustaram melhor ao modelo linear. Observou-se também que sementes armazenadas por 3 e 6 meses, quando colocadas para germinar em solução-controle, apresentaram maior velocidade de germinação do que aquelas recém-colhidas (mês 0). Avaliando-se os

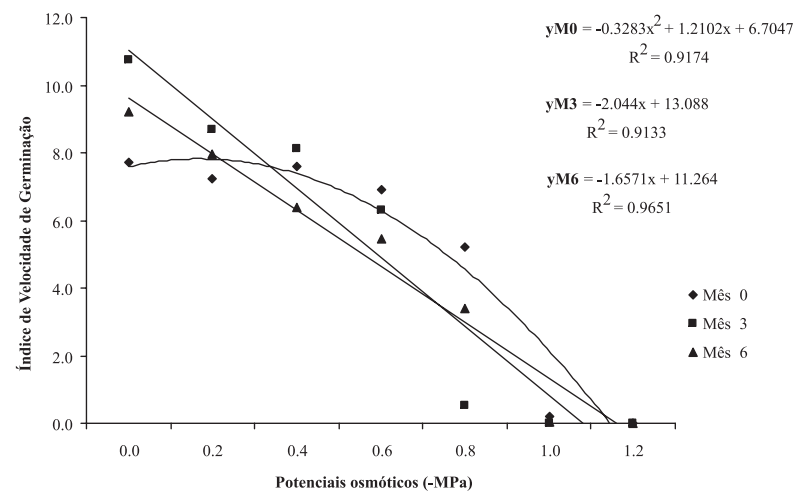

Figura 5 - Índice de Velocidade de Germinação (IVG) das sementes de Caesalpinia pyramidalis Tul. submetidas a diferentes tratamentos de deficiência hídrica (-MPa) e armazenadas por 0, 3 e 6 meses em temperatura ambiente e saco de papel kraft. Feira de Santana, Bahia, agosto de 2007 a fevereiro de 2008.

Figure 5 - Germination speed rate of Germination (GSR) of Caesalpinia pyramidalis Tul. seeds subjected to differents treatments of water deficiency $(-\mathrm{MPa})$ and stored for 0,3 and 6 months in room temperature and kraft paper bag. Feira de Santana, Bahia, August 2007 to February 2008.

resultados relativos ao Índice de Velocidade de Germinação em sementes de catingueira, pôde-se inferir que o vigor das sementes é mantido em estresse moderado, decrescendo quando há deficiência hídrica mais intensa.

Resultados similares foram encontrados por outros autores, como Souza Filho (2000), que, estudando o comportamento das sementes de Leucena leucocephala sob déficit hídrico (simulado com o uso de soluções de polietilenoglicol), observou que o IVG das sementes foi afetado a partir de potenciais osmóticos mais reduzidos (-0,9 MPa); Ribeiro (2008), a qual observou que o vigor das sementes da leguminosa G. sepium armazenadas por 6 meses em laboratório, tendeu a reduzir com a diminuição do potencial osmótico; Farias et al. (2009), que verificaram que o IVG das sementes de G. sepium tendeu a decrescer à medida que o potencial osmótico tornou-se mais negativo, já que as sementes necessitam de mais tempo para embeber e germinar, evidenciando menor velocidade de germinação; e Bakke et al. (2006), que observaram uma significativa redução nos valores de IVG das sementes de juremapreta (Mimosa tenuiflora) com o aumento do estresse hídrico proporcionado pelo PEG 6000. 


\section{CONCLUSÃO}

Os resultados obtidos com as sementes de catingueira são promissores em estudos voltados ao envigoramento de sementes, os quais necessitam da determinação do potencial de priming, a partir de ensaios que envolvem deficiência hídrica em sementes.

A identificação dos fatores ambientais que governam o processo germinativo das sementes, tal como a disponibilidade de água para as mesmas, representa uma importante ferramenta na interpretação do comportamento ecológico das espécies sob condições de habitats naturais.

\section{AGRADECIMENTOS}

Ao CNPq pelo apoio financeiro e À Embrapa/SemiÁrido, Petrolina, PE, especialmente na pessoa da Dra. Bárbara Aragão, pelo fornecimento de material vegetal.

\section{REFERÊNCIAS}

AMBIENTE BRASIL. Classificação climática de Köppen-Geiger. 2005. Disponível em: <www.ambientebrasil.com.br> . Acesso em: 30/06/08.

BABU, R. C. et al. Comparison of measurement methods of osmotic adjustment in rice cultivars. Crop. Science, v.39, n.1, p.150-158, 1999.

BAKKE, I. A. et al. Water and sodium chloride effects on Mimosa tenuiflora (WILLD.) POIRET seed germination. Revista Caatinga, v.19, n.3, p.261-267, 2006.

BANSAL, R. P.; BHATI, P. R.; SEN, D. N. Differential specificity in water inhibition of Indian arid zone. Biologia Plantarum, v.22, n.2, p.327-331, 1980.

BARBEDO, C. J.; MARCOS-FILHO, J. Tolerância à dessecação de sementes. Acta Botânica Brasílica, v.12, n.2, p.145-164, 1998.

BEWLEY, J. D.; BLACK, M. Physiology and biochemistry of seeds. 2.ed. New York: Springer Verlag, 1982. 375p.

BEWLEY, J. D.; BLACK, M. Seeds: physiology of development and germination. New York: Plenum Press, 1985. 367p.
BEWLEY, J. D.; BLACK, M. Seeds: physiology of development and germination. 2.ed. New York: Plenum Press, 1994. 445p.

BLAKE, T. J. Transplanting shock in white spruce: Effect of cold storage and root pruning on water relations and stomatal conditioning. Plant Physiology, v.57, n.2, p.210-216, 1993.

BORGES, E. E. L.; RENA, A. B. Germinação de sementes. In: AGUIAR, I. B.; PIÑA-RODRIGUES, F. C. M.; FIGLIOLIA, M. B. Sementes florestais tropicais. Brasília: ABRATES, 1993. p.133-135.

BRACCINI, A. L. et al. Germinação e vigor de sementes de soja sob estresse hídrico induzido por soluções de cloreto de sódio, manitol e polietilenoglicol. Revista Brasileira de Sementes, v.18, n.1, p.10-16, 1996.

CARVALHO, C. J. R. Respostas de plantas de Schizolobium amazonicum [S. parahyba var. amazonicum] e Schizolobium parahyba [Schizolobium parahybum] à deficiência hídrica. Revista Árvore, v.29, n.6, p.907-914, 2005.

CARVALHO, N.M.; NAKAGAWA, J. Sementes: ciência, tecnologia e produção. 4ed. Jaboticabal: FUNEP, 2000, 588p

CAVAlCANTE, A. M. B.; PEREZ, S. C. J. G. A. Efeitos dos estresses hídrico e salino sobre a germinação de sementes de Leucaena leucocephala (Lam.) de Wit. Pesquisa Agropecuária Brasileira, v.30, n.2, p.281-289, 1995.

DANTAS, J. P. et al. Avaliação de genótipos de feijão-de-corda sob salinidade. Revista Brasileira de Engenharia Agrícola e Ambiental, v.6, n.3, p.425-430, 2002.

FARIAS, S. G. G. et al. Efeitos dos estresses hídrico e salino na germinação de sementes de gliricidia [Gliricidia sepium (Jacq.) Steud.]. Revista Caatinga, v.22, n.4, p.152-157, 2009.

FERREIRA, D. F. Sisvar 4. 3 - Sistema de análises estatísticas. Lavras: Universidade Federal de Lavras, 1999.

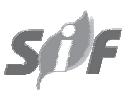

Revista Árvore, Viçosa-MG, v.35, n.5, p.1007-1015, 2011 
FERREIRA, A. G.; BORGHETTI, F.

Germinação: do básico ao aplicado. São Paulo: Artmed, 2004. 323p.

FONSECA, S. C. L.; PEREZ, S. C. J. G. A. Ação do polietileno glicol na germinação de sementes de Adenanthera pavonina l. e o uso de poliaminas na atenuação do estresse hídrico sob diferentes temperaturas. Revista Brasileira de Sementes, v.25, n.1, p.1-6, 2003.

FOOLAD, M. R. Recent advances in genetics of salt tolerance in tomato. Plant Cell, v.76, n.2, p. 101-119, 2004.

FRANCELINO, M. R. et al. Contribuição da caatinga na sustentabilidade de projetos de assentamentos no sertão norte-rio-grandense. Revista Árvore, v.27, n.1, p.79-86, 2003.

HADAS, A. Water uptake and germination of leguminous seeds under changing external water potential in osmotic solutions. Journal of Experimental Botany, v.27, n.98, p.480-489, 1976.

HARDEGREE, S. P.; EMMERICH, W. E. Effect of polyethylene glycol exclusion on the water potential of solution saturated filter paper. Plant Physiology, v.92, n.2, p.462-466, 1990.

HASEGAWA, P. M. et al. Cellular mechanisms of tolerance to water stress. Hortscience, v.19, n.3, p.371-377, 1984.

JELLER, H.; PEREZ, S. C. J. G. A. Condicionamento osmótico na germinação de sementes de cássia-do-nordeste sob estresse hídrico, térmico e salino. Pesquisa

Agropecuária Brasileira, v.38, n.9, p.1025-1034, 2003.

MONTEIRO, J. M. et al. Teor de taninos em três espécies medicinais arbóreas simpátricas da caatinga. Revista Árvore, v.29, n.6, p.999-1005, 2005.

MORAES, G. A. F.; MENEZES, N. L. Desempenho de sementes de soja sob condições diferentes de potencial osmótico. Ciência Rural, v.33, n.2, p.219-226, 2003.
NASSIF, S. M. L.; PEREZ, S. C. J. G. A. Germinação de sementes de amendoim-do-campo (Pterogyne nitens Tul. - FabaceaeCaesalpinoideae) submetidas a diferentes condições de estresse hídrico e salino. Revista Brasileira de Sementes, v.19, n.2, p.142-149, 1997.

PEREZ, S. C. J. G. A.; FANTI, S. C.; CASALI, C. A. Influência da luz na germinação de sementes de canafístula submetidas ao estresse hídrico. Bragantia, v.60, n.3, p.155-166, 2001.

RIBEIRO, R. C. Efeito do armazenamento na qualidade fisiológica de sementes de Gliricidia sepium (Jacq.) Steud. (Leguminosae - Papilionoideae). 2008. 100f. Dissertação (Mestrado em Botânica) Universidade Estadual de Feira de Santana, Feira de Santana, 2008.

SAMPAIO, E. V. S. B. Overview of Brazilian caatinga. In: MOONEY, H. A.; BULLOCK, S. H.; MEDINA, E. Dry tropical forests.

Cambridge: Cambridge University Press, 1995. p.35-63.

SAMPAIO, E. V. S. B.; RODAL, M. J. N. Fitofisionomia da Caatinga. In: Avaliação e identificação de ações prioritárias para a conservação, utilização sustentável e repartição de benefícios da biodiversidade do bioma Caatinga. Petrolina: 2000. p.2-14. (Documento para discussão no GP Botânica).

SANTOS, V. L. M. et al. Efeito do estresse salino e hídrico na germinação e vigor de sementes de soja. Revista Brasileira de Sementes, v.14, n.2, p.189-194, 1992.

SOUZA FILHO, A. P. S. Influência da temperatura, luz e estresses osmótico e salino na germinação de sementes de Leucaena leucocephala. Pasturas Tropicales, v.22, n.2, p.47-53, 2000.

TAYLOR, A. G.; HARMAN, G. E. Concepts and technologies of selected seed treatments.

Annual Review Phytopathlogy, v.28, p.321-339, 1990. 
VERSLUES, P. E. et al. Methods and concepts in quantifying resistance to drought, salt and freezing, abiotic stresses that affect plant water status. The Plant Journal, v.45, n.4, p.523-539, 2006.

VILLELA, F. A.; DONI FILHO, L.; SEQUEIRA, E. L. Tabela de potencial osmótico em função da concentração de polietilenoglicol 6000 e da temperatura. Pesquisa Agropecuária Brasileira, v.26, n. 11/12, p.1957-1968, 1991.
WOODSTOCK, L. W.; TAO, K. L. J. Prevention of imbibitional injury in low vigor soybean embryonic axes by osmotic control of water uptake. Physiologia Plantarum, v.51, n .1, p.133-139, 1981.

ZHU, J. K. Salt and drought stress signal transduction in plants. Annual Review of Plant Biology, v.53, p.247-273, 2002. 
